\title{
In vivo functional photoacoustic imaging of brain tumor vasculature
}

Jung-Taek Oh, Meng-Lin Li, Xueyi Xie, George Stoica, Lihong V. Wang

Jung-Taek Oh, Meng-Lin Li, Xueyi Xie, George Stoica, Lihong V. Wang, "In vivo functional photoacoustic imaging of brain tumor vasculature," Proc. SPIE 6086, Photons Plus Ultrasound: Imaging and Sensing 2006: The Seventh Conference on Biomedical Thermoacoustics, Optoacoustics, and Acoustooptics, 60860C (6 March 2006); doi: 10.1117/12.645391

SPIE. Event: SPIE BiOS, 2006, San Jose, California, United States 


\title{
In vivo functional photoacoustic imaging of brain tumor vasculature
}

\author{
Jung-Taek Oh, Meng-Lin Li, Xueyi Xie \\ Optical Imaging Laboratory, Department of Biomedical Engineering, Texas A\&M University, TX \\ 77843-3120 \\ George Stoica \\ Department of Pathobiology \\ Texas A\&M University, College Station, TX 77843-5547 \\ Lihong V. Wang* \\ Optical Imaging Laboratory, Department of Biomedical Engineering, Texas A\&M University, TX \\ $77843-3120$
}

\begin{abstract}
We present a study of the functional photoacoustic imaging of tumor hypoxia in mice in vivo. Based on spectroscopic photoacoustic tomography that detects the optical absorption of oxy- and deoxy-hemoglobins, the blood oxygen saturation and the vascularization of brain tumors were visualized. U87 glioblastoma tumor cells were inoculated intracranially at a 3-mm depth from the surface of the nude mouse head seven days before the experiment. Increased blood content and hypoxia inside the tumor vasculature were detected through the intact skin and skull. This technique will be useful for future studies on tumor metabolic activities in the brain and hypoxia-related therapeutic resistance.
\end{abstract}

Keywords: photoacoustic tomography, tumor hypoxia, spectroscopic imaging, blood oxygenation

\section{INTRODUCTION}

Tumor hypoxia is important in tumor biology and therapy ${ }^{1,2}$. Hypoxia in solid tumors occurs as a result of an inadequate supply of oxygen due to exponential cellular proliferation or an inefficient vascular supply. ${ }^{1}$ Hypoxia inhibits cell reproduction and can even induce cell death, but it also provides angiogenic and metastatic signals, which allow prolonged survival in the absence of oxygen.

Studies of tumor vasculature in terms of oxygen supply and blood volume have provided understanding of tumor oxygen transport, which helps to assess hypoxia in tumors. Tumor hypoxia is known to accompany low oxygen saturation levels in the microvasculature around tumors. Recently, the hypoxic state in microvasculature was imaged by a hyperspectral imaging method with an invasive window chamber. ${ }^{3}$

Although several technologies have been previously explored as noninvasive tumor hypoxia and tumor vasculature imaging methods, almost all of them suffer from critical limitations. Contrast-enhanced dynamic computerized tomography for tumor perfusion imaging requires a contrast agent and is incapable of providing an accurate estimate of tumor oxygenation ${ }^{4}$. Electron paramagnetic resonance (EPR) spectroscopy has been used to determine the oxygenation

\footnotetext{
* Corresponding author: LWang@tamu.edu.
} 
status of tumors $10 \mathrm{~mm}$ from the surface of the body by using stable nitroxide-free radical probes which have not yet been fully tested in human patients. ${ }^{5}$ Oxygen dependent quenching of phosphorescence is another non-invasive optical method for measuring oxygen pressure in tissues. ${ }^{6}$ However, this method employs phosphors, which have not previously been used clinically in human patients, plus it is difficult to obtain a good signal-to-noise-ratio. Diffuse optic tomography can also be used to detect oxygen saturation utilizing the intrinsic absorption spectrum of hemoglobin. ${ }^{7}$ The resolution and accuracy is limited, however, by the light scattering of the biological tissues when the imaging of the brain is conducted through the intact skin and skull.

Functional photoacoustic tomography is a new non-invasive imaging modality, and it is emerging as a very practical method for imaging biological tissue structures by means of laser-induced ultrasound. ${ }^{8}$ Structures with high optical absorption, such as blood vessels, can be imaged with the spatial resolution of ultrasound, which is not limited by the strong light scattering in biological tissues. By varying the wavelengths of the laser light and acquiring photoacoustic images, the optical absorption spectrum of each image pixel is found. Since the biochemical constituents of tissues are what determine the absorption spectrum, useful functional information, like the oxygen saturation $\left(\mathrm{SO}_{2}\right)$ and the total haemoglobin concentration $(\mathrm{HbT})$, can be extracted. In addition, the oxygen saturation of the vasculature in the brain cortex has been successfully measured by functional photoacoustic tomography. ${ }^{9}$

In our study, we used functional photoacoustic tomography to image the hypoxic brain tumor vasculature. High resolution brain vasculature images of the oxygen saturation and the total hemoglobin concentration were used to visualize the hypoxic tumor vasculature. Because of the location of the tumor inside the brain, conventional optical imaging modalities are not able to detect in vivo vasculature information with sub $\mathrm{mm}$ spatial resolution. Here, brain tumor cells were inoculated, and the tumor positions and hypoxia were identified by the low $\mathrm{SO}_{2}$ and the high $\mathrm{HbT}$ levels of the vasculature in the images. The results were compared with conventional histopathologies to confirm that the abnormal vasculature of the brain coincided with the tumor positions. Statistical differences between the normal and the tumor sites were found in all three animal samples $(n=3)$.

\section{MATERIAL AND METHODS}

\subsection{Experimental setup}

The experimental setup for the functional photoacoustic tomography of a brain tumor in vivo is shown in Figure 1 . A tuneable Ti:Sa nanosecond pulse laser (LT-2211A, Lotis T II, Minsk, Belarus) pumped by an Nd:YAG laser (LS2137/2, Lotis T II, Minsk, Belarus) was employed to provide laser pulses with a pulse repetition rate of $10 \mathrm{~Hz}$. The laser beam was expanded by a light diffuser and then delivered to the animal head. The incident energy density of the laser beam on the surface of the mouse head was controlled at $\sim 30 \mathrm{~mJ} / \mathrm{cm} 2$ (within the ANSI standard). The laser pulse energy was measured with a photodiode, and the measured energy was used to compensate for fluctuations in the laser output and the corresponding photoacoustic signals. Ultrasonic transducers (V323/2.25MHz, Panametrics, 50\% bandwidth) were used as detectors to receive the induced acoustic signals. The large active area (6 mm diameter) of the transducer increased the sensitivity. Four wavelengths, 764, 784, 804 and $824 \mathrm{~nm}$, were adopted in each scanning step. A step motor drove the transducers to scan circularly with a step size of 3 degrees. The mouse was held by a homemade fixture with its head protruding into the water tank through a hole in the bottom. The hole was sealed with a piece of thin plastic film. The mouse head surface was covered with a thin layer of ultrasonic coupling gel. The detected photoacoustic signals were amplified and then digitized by a Gage PC card (CS14100, Gage). The distribution of the optical absorption in the imaging plane (x-y plane) was reconstructed using a modified back-projection algorithm. ${ }^{10}$ The measured spatial resolution was $0.26 \mathrm{~mm}$ from the FWHM of the point spread function.

\subsection{Theory}

Images from four wavelengths of the laser light source were processed to create $\mathrm{HbT}$ and $\mathrm{SO}_{2}$ maps. The method for determining both $\mathrm{HbT}$ and $\mathrm{SO}_{2}$ in the vasculature from photoacoustic images was based on the following model: ${ }^{9}$ 
Wave length tuning $(\lambda: 764,784,804,824 \mathrm{~nm})$

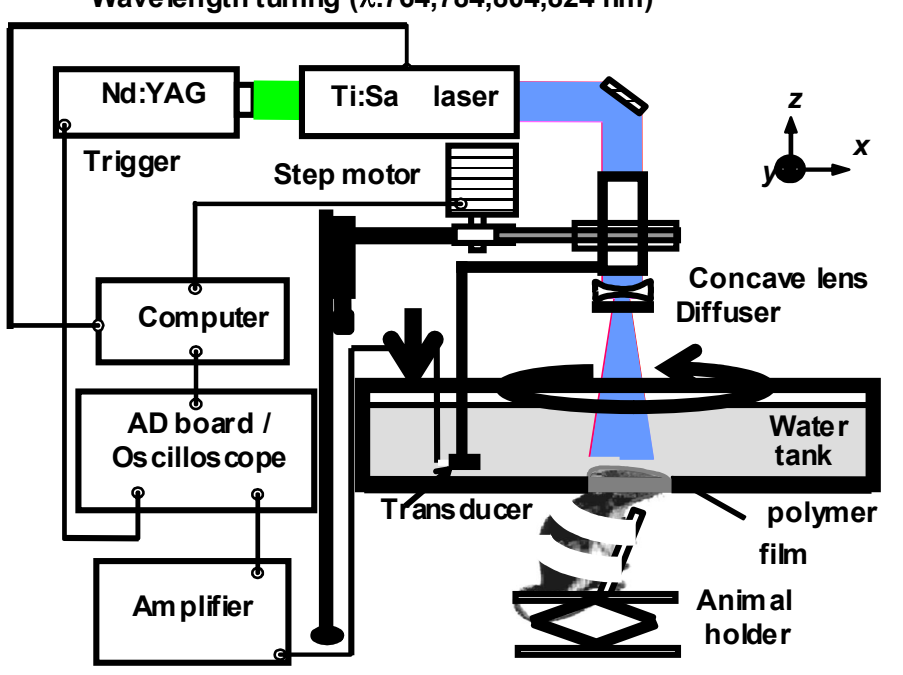

Figure 1. Schematic of the functional PAT system for imaging of the total concentration of hemoglobin and the hemoglobin oxygen saturation in a rat brain in vivo.

$$
P A_{\lambda}=k \varepsilon_{\lambda}^{H b}[\mathrm{Hb}]+k \varepsilon_{\lambda}^{\mathrm{HbO}_{2}}\left[\mathrm{HbO}_{2}\right]
$$

where $\mathrm{PA}_{\lambda}$ is the pixel value of a reconstructed photoacoustic image at the given wavelength $\lambda$; $\varepsilon_{\lambda}{ }^{\mathrm{Hb}}$ and $\varepsilon_{\lambda}{ }^{\mathrm{HbO} 2}$ are the molar extinction coefficient for the deoxy- and oxy-haemoglobin for the wavelength $\lambda$, respectively, $[\mathrm{Hb}]$ and $\left[\mathrm{HbO}_{2}\right]$ are the molar concentration of deoxy- and oxyhemoglobin, repectively, and $\mathrm{k}$ is the scaling constant. In this model, it is assumed that $\mathrm{Hb}$ and $\mathrm{HbO}_{2}$ are the main absorbers in the living tissues in the 764 to 824 nm wavelength range, and that the light can penetrate the sample volume by the weak absorption. [Hb] and $\left[\mathrm{HbO}_{2}\right]$ are estimated with the linear leastsquares of multi-wavelength $\mathrm{PA}_{\lambda}$ values and the linear model in Eq. (1). $\mathrm{SO}_{2}$ and $\mathrm{HbT}$ are calculated from the following equations:

$$
\begin{aligned}
\mathrm{HbT} & =\left[\mathrm{HbO}_{2}\right]+[\mathrm{Hb}] \\
\mathrm{SO}_{2} & =\frac{\left[\mathrm{HbO}_{2}\right]}{\left[\mathrm{HbO}_{2}\right]+[\mathrm{Hb}]}
\end{aligned}
$$

\subsection{In-vitro blood test}

The functional PAT system was applied to measuring the $\mathrm{SO}_{2}$ levels of bovine blood samples in vitro. A blood sample was collected from a bovine subject, and high and low $\mathrm{SO}_{2}$ levels of blood were prepared by exposing the blood to $\mathrm{O}_{2}$ and $\mathrm{CO}_{2}$, respectively. These two blood samples were mixed with different volume ratios to achieve various $\mathrm{SO}_{2}$ levels ranging from $29 \%$ to $93 \%$, and were then measured by a NIR spectrophotometer after sampling a small amount and hemorrhaging the red blood cell using the saporin. ${ }^{11}$ Mixed blood samples were injected into $0.25 \mathrm{~mm}$ inner-diameter optically and acoustically transparent tubes buried inside the chicken breast tissue phantom (thickness $=2 \mathrm{~mm}$ ). Three tubes were placed in the same plane and separated by around $2 \mathrm{~mm}$. The three tubes with different $\mathrm{SO}_{2}$ level blood samples were imaged at the same time. The measured $\mathrm{SO}_{2}$ level from the functional PAT images were linear to the spectrophotometer results with a high linear regression coefficient $\mathrm{R}=0.99$. However, there was a systematic bias in the measurement results because of an overestimation of the deoxy-haemoglobin. Calibration results from previous the invitro blood tests were therefore applied. 


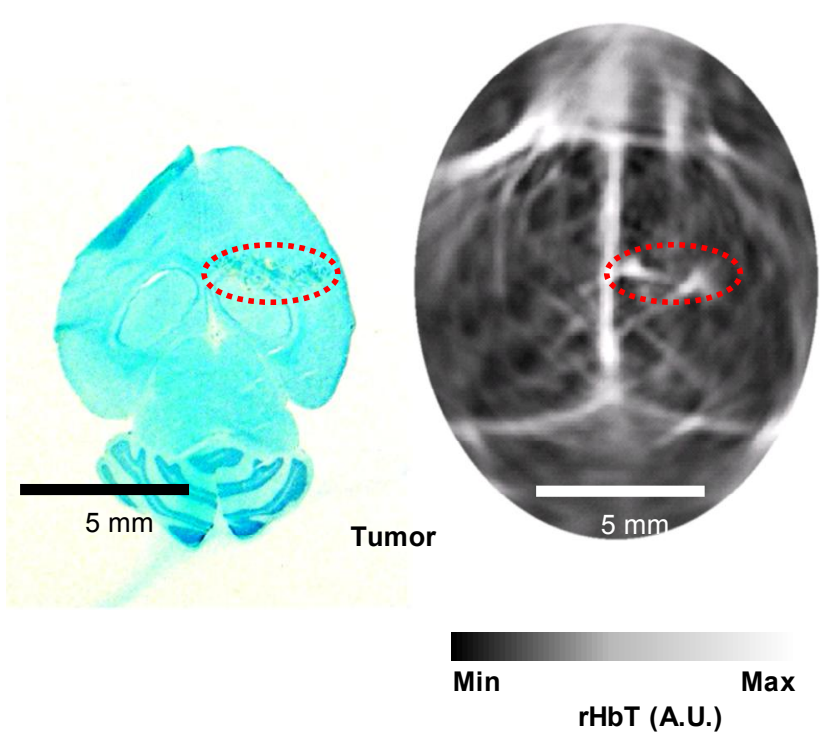

(a) (b)
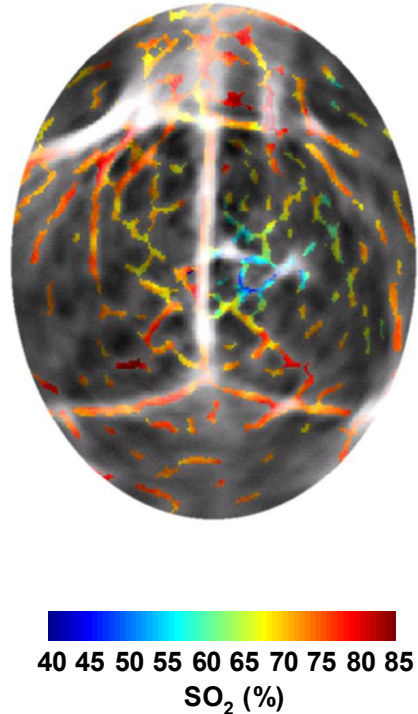

(c)

Figure 2. Non-invasive functional photoacoustic image of the total concentration of hemoglobin ( $\mathrm{HbT}$ ) and the hemoglobin oxygen saturation $\left(\mathrm{SO}_{2}\right)$ inside a nude mouse brain with $\mathrm{U} 87$ cell lines. (a) microslide cross section colored with Thionine staining. (b) and (c) images of relative $\mathrm{HbT}$ and $\mathrm{SO} 2$ inside a nude mouse brain.

\subsection{Animal models}

U87 glioblastoma tumour cells were used for inoculation into young adult immunocompromised nude mice (Harlan, Co.). Solid glioblastomas are known to create hypoxia inside a tumor. ${ }^{12}$ The cells $\left(1 \times 10^{6}\right)$ were inoculated intracranially into the caudate nucleus at $3 \mathrm{~mm}$ depth from the mouse head surface with a volume of $7 \mu \mathrm{l}$ by using a $10 \mu 1$ Hamilton syringe. Stereotactic implantation of the U87 glioblastoma tumour cells was performed, under full anaesthesia, on nude mice weighing about 20 grams using a mixture of ketamine, $87 \mathrm{mg} / \mathrm{kg}$ (Ketaset, Forth Dodge Animal Health) and xylazine, $13 \mathrm{mg} / \mathrm{kg}$ (AnaSed, Lloyd Laboratories). Solid tumors were allowed to develop inside the brain for five days following inoculation with the tumor cells. On the sixth day, the nude mice were imaged by the functional photoacoustic tomography system.

Before imaging, a dose of $87 \mathrm{mg} / \mathrm{kg}$ Ketamine plus Xylasine $13 \mathrm{mg} / \mathrm{kg}$ was administered intramuscularly, was used to anesthetize the mice, and supplemental injections of a similar anesthetic mixture $(\sim 50 \mathrm{mg} / \mathrm{kg} / \mathrm{hour})$ kept them motionless throughout the experiment. Inhaled pure oxygen gas was ventilated to the mice at a flow rate of $\sim 0.6 \mathrm{l} / \mathrm{min}$. During the experiment, the sensor of a Pulse Oximeter ( $8600 \mathrm{~V}$, Nonin Medical, Inc.) clamped the back paw of the mouse to monitor the global arterial blood oxygenation. The nude mouse body temperature was controlled at $37^{\circ} \mathrm{C}$ using a water heating pad. After the experiments, the mice were sacrificed to obtain brain section slides. The excised samples were stained with Thionine, which is known to color small animal brain tumors dark blue.

All the performed experimental animal procedures on the nude mice were approved by the University Laboratory Animal Care Committee of Texas A\&M University and followed the guidelines of the United States National Institutes of Health (Guide for the Care and Use of Laboratory Animals, NIH Publication No. 86-23, revised 1985. US Government Printing Office, Washington DC). 


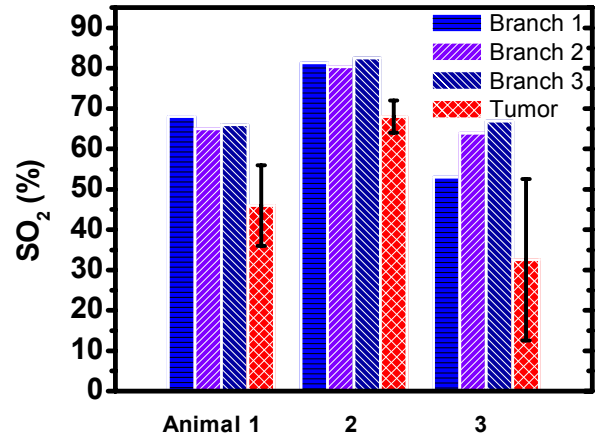

(a)

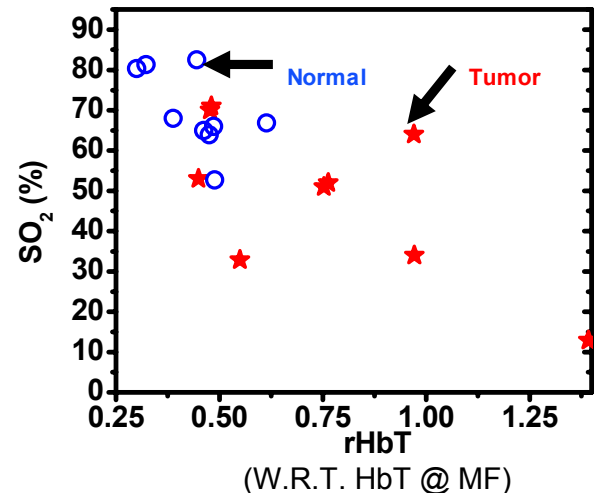

(b)

Figure 3. Statistical results extracted from the functional photoacuostic tomography images of the brain tumor vasculature. (a) Hemoglobin oxygen saturation (SO2) distribution for both normal and the tumor vasculature. (b) Total concentration of hemoglobin versus oxygen saturation for both normal and tumor vasculature for all animals $(\mathrm{n}=3)$.

\section{RESULTS}

Functional photoacoustic images of the mouse brain with a hypoxic U87 glioblastoma tumor are shown in Figure 2 along with the corresponding histology. The Thionine-stained microslide imge in Figure 2(a) indicates a tumor at the middle of the right hemisphere of the brain. At the same anatomical position as the tumor in the $\mathrm{HbT}$ image, in Figure 2(b), 3 to 4 times higher $\mathrm{HbT}$ values than normal vasculature are observed. The $\mathrm{SO}_{2}$ image in Figure 2(c) also indicates an abnormal distribution of oxygen level at the same position. The difference in $\mathrm{SO}_{2}$ between the hypoxic tumor vasculature and the normal blood vessels is more than $20 \%$ in Figure 2. Also, the $\mathrm{SO}_{2}$ image shows a larger area of hypoxia than the $\mathrm{HbT}$ and microslide images.

Three animals $(n=3)$ were imaged in this study in order to have statistically significiant results. We chose three healthy blood vessels and three examples of tumor vasculature from each object to compare normal and tumor vasculature in the images. Figure 3(a) is a plot of the $\mathrm{SO}_{2}$ levels in the healthy vessels and the tumor vasculature. It shows that the difference in the mean $\mathrm{SO}_{2}$ level from the two tissues is more than $20 \%$ for all animal objects. The heterogeneity of the $\mathrm{SO}_{2}$ level is stronger in the tumor vasculature than in the normal vessels inside the brain. In this experiment, the intraanimal comparison is difficult because the overall oxygen saturation rate varies due to different inhalation conditions and the depth at which the anaesthesia affects the brain $\mathrm{SO}_{2}$ level. The observed characteristics of low oxygen level and large fluctuations in the tumor vasculature are manifested by strong $\mathrm{HbT}$ surrounding it. Figure 3(b) shows the relationship between the $\mathrm{HbT}$ and $\mathrm{SO}_{2}$ values for both tumorous and healthy vasculature. The trend for healthy vessels is low $\mathrm{HbT}$ and high $\mathrm{SO}_{2}$ while hypoxic tumor vasculature has high $\mathrm{HbT}$ and low $\mathrm{SO}_{2}$ with large fluctuations in these values.

\section{DISCUSSION}

Non-invasive tumor vasculature imaging may expand our understanding of tumor physiology and help in the development of new tumor treatments. Here we were interested in quantifying the hemoglobin oxygenation and the hemoglobin concentration in the hypoxic brain tumor vasculature non-invasively through the intact skin and skull. To this end, we have developed a functional photoacoustic tomography system and provided proof-of-principle experiments to demonstrate the the effectiveness of the system for imaging tumor vasculature.

In the previous studies, Sorg et al. have used a hyperspectral imaging system to measure hemoglobin oxygenation in the microvasculature of tumors imbedded inside a window chamber. ${ }^{3}$ Since the method was based on reflectance type microscopy that is limited by the light scattering, direct exposure of the tumors is necessary. Microvasculature imaging 
results indicated low oxygen saturation levels in the middle of the hypoxic tumor, which was identified by hypoxiadriven green florescence protein (GFP).

Figure 2 shows proof-of-principle experiments measuring deep tumors inside the brain, which are inaccessible to conventional oxygen saturation imaging modalities. Higher blood content levels were observed in the tumor vasculature, which was identified by the histopathology. The increased HbT level was not an unexpected result. Prior researches on tumor detection methods based on the blood absorption showed the same trend. Wide varieties of tumors have been reported to have increased blood content, which is expressed in high HbT values. ${ }^{13}$ Although the tumor cell line in this research (U87 glioblastoma) has not been examined with other modalities exclusively, we can accept the findings of the functional phatoacoustic images, given the fact that solid tumors induce angiogenesis to supply the additional blood required for their upregulated metabolism. ${ }^{1}$ Our study shows a hypoxic state as well as heterogeneity in the oxygen saturation level in the tumor vasculature. Based on the facts that developed U87 tumors induce hypoxia inside solid tumors ${ }^{12}$ and that low $\mathrm{SO}_{2}$ levels exist in the microvasculature near hypoxia tumors, we can conclude that functional photoacoustic tomography can detect oxygen delivery states at the tumor site non-invasively with no biological modifications. As a next step, we need to correlate tumor hypoxia and a low $\mathrm{SO}_{2}$ level in the vasculature of the same animal at the same imaging point. Previously, Sorg et al. used the GFP to correlate both pieces of information and confirm this correlation. ${ }^{3}$ Once a hypoxia-driven protein can be synthesized to bind with a contrast agent sensitive to photoacoustic tomography, the full relationship between tumor hypoxia and the vasculature oxygen level will be investigated to provide additional understanding of tumor physiology.

\section{CONCLUSION}

In this study, hypoxic brain tumor vasculature was imaged with functional photoacoustic tomography. In-vivo and noninvasive high resolution brain vasculature images of the oxygen saturation and the total hemoglobin concentration was provided to visualize the hypoxic tumor vasculature. Hypoxic tumors were developed inside the mouse brain, and the tumor positions and hypoxia were identified by the low oxygen saturation and the high total hemoglobin concentration of the vasculature. Conventional histopathology confirmed that the abnormal sites in the brains (in terms of vasculature) coincided with the tumor position. Statistical differences between the normal and tumor sites showed clear distinctions. However, the correlation between low $\mathrm{SO}_{2}$ level in the vasculature and tumor hypoxia must be clearly established for tumor hypoxia detection, since functional photoacoustic tomography images only the vasculature inside the brain. Molecular imaging or gene expression imaging techniques in photoacoustic tomography may be deployed to verify the correlation. The demonstrated functional photoacoustic tomography technique can be useful for future studies on tumor metabolic activities in the brain and hypoxia-related therapeutic resistance.

\section{REFERENCES}

1. J.M. Brown, A.J. Giaccia, "The unique physiology of solid tumors: opportunities (and problems) for cancer therapy," Cancer Res. 58, pp. 1408-1416 (1998).

2. P. Vaupel ,M. Hockel, "Tumor oxygenation and its relevance to tumor physiology and treatment," Adv. Exp. Med. Biol. 510, pp. 45-49 (2003).

3. B. Sorg, B. Moeller, O. Donovan, Y. Cao, and M. Dewhirst, "Hyperspectral imaging of hemoglobin saturation in tumor microvasculature and tumor hypoxia development," J. Biomed. Opt. 10, 044004 (2005).

4. R. Hermans, P. Lambin, A. Van der Goten, W. Van den Bogaert, B. Verbist, C. Weltens ,P.R. Delaere, "Tumoural perfusion as measured by dynamic computed tomography in head and neck carcinoma," Radiother Oncol. 53, pp.105111 (1999).

5. H. Swartz ,R.B. Clarkson, The measurement of oxygen in vivo using EPR techniques, Phys. Med. Biol. 43, pp. 19571975 (1998).

6. D. Wilson, S. Vinogradov, L.W. Lo ,L. Huang, Oxygen dependent quenching of phosphorescence: a status report, Adv. Exp. Med. Biol. 388, pp. 101-107 (1996). 
7. C. Cheung, J.P. Culver, K. Takahashi, J.H. Greenberg ,A.G. Yodh, In vivo cerebrovascular measurement combining diffuse near-infrared absorption and correlation spectroscopies, Phys. Med. Biol. 46, pp. 2053-2065 (2001).

8. X. Wang, Y. Pang, G. Ku, X. Xie, G. Stoica, and L. Wang, "Noninvasive laser-induced photoacoustic tomography for structural and functional in vivo imaging of the brain," Nat, Biotechnol. 21, 803-806 (2003).

9. X. Wang, X. Xie, G. Ku, G. Stoica, and L. Wang, "Functional photoacoustic tomography for non-invasive imaging of cerebral blood oxygenation and blood volume in rat brain in vivo," SPIE 5697, pp.1-6 (2005).

10. M. Xu, Y. Xu, and L. Wang, "Time-domain reconstruction algorithms and numerical simulations for thermoacoustic tomography in various geometries," IEEE Trans. on Biomed. Eng. 50, pp.1086-1099 (2003).

11. P. Scheid, and M. Meyer, "Mixing technique for study of oxygen-haemoglobin equilibrium: a critical evaluation," $J$. Appl. Physiol. 45, 818-822 (1978).

12. P. Wachsberger, R. Burd, N. Marero, C. Daskalakis, A.Ryan, P. McCue and A. Dicker "Effect of the Tumor Vascular-Damaging Agent, ZD6126, on the Radioresponse of U87 Glioblastoma," Cli. Cancer Res. 11, pp. 835-842 (2005).

13. D. Grosenick, H. Wabnitz, K. Moesta, J. Mucke, M. Moller, C. Stroszczynski, J, Stobel, B. Wassermann, P. M Schlag, and H, Rinneberg, "Concentration and oxygen saturation of haemoglobin of 50 breast tumors determined by time-domain mammography," Phys. Med. Biol. 49, pp.1165-1181 (2004). 